\title{
Consistent Extension of Quasidilaton Massive Gravity
}

\author{
Josef Klusoň \\ Department of Theoretical Physics and Astrophysics, Faculty of Science, Masaryk University, Kotlárská 2, 61137 Brno, Czech Republic
}

Correspondence should be addressed to Josef Klusoň; klu@physics.muni.cz

Received 22 May 2014; Accepted 4 July 2014; Published 20 July 2014

Academic Editor: Sergey D. Odintsov

Copyright (C) 2014 Josef Klusoň. This is an open access article distributed under the Creative Commons Attribution License, which permits unrestricted use, distribution, and reproduction in any medium, provided the original work is properly cited.

\begin{abstract}
This paper is devoted to the Hamiltonian analysis of extension of the quasidilaton massive gravity as was proposed recently in [arXiv:1306.5502]. We show that, for given formulation of the theory, the additional primary constraint that is responsible for the elimination of the Boulware-Deser ghost is missing. We compare this situation with the quasidilaton massive gravity. Finally, we propose the ghost-free extension of quasidilaton massive gravity.
\end{abstract}

\section{Introduction and Summary}

Recently, new version of the full nonlinear massive gravity was found by de Rham, Gabadadze and Toley (dRGT) $[1,2]$ that provides the positive answer to the question of whether graviton can have a nonzero mass. In fact, among many remarkable properties, there is the crucial one which is the absence of the Boulware-Deser ghost $[3,4]$.

The consistent massive gravity could also provide a possible explanation of the observed acceleration of the cosmic expansion which is one of the greatest mysteries in modern cosmology. It is tempting to speculate that the finite graviton mass could be a source of the accelerated expansion of the universe. For that reason, it is of great interest to formulate theoretically consistent cosmological scenario in massive gravity that is also in agreement with the observations. Unfortunately, it was recently shown that all homogeneous and isotropic cosmological solutions in dRGT theory are unstable [5]; see also [6-8].

In order to resolve this problem, we have two possible options: either to break homogeneity [9] or isotropy [10,11] or to extend the theory as in $[12,13]$. Recently in [14], de Felice and Mukohyama proposed new extension of the quasidilaton massive gravity that could provide stable and self-accelerating homogeneous and isotropic cosmological solution. They further argued that given extension belongs to the class of models studied in [15] that are free from the Boulware-Deser ghosts. However, the explicit Hamiltonian analysis of given theory was not performed in [14].
The goal of this paper is to reconsider the problem of the Boulware-Deser ghost in the model [14]. We present an evidence that, for the action that was introduced in [14], the Boulware-Deser ghost cannot be eliminated. More precisely, performing the Hamiltonian analysis of this model with the time dependent quasidilaton, we find that the primary constraint which is responsible for the elimination of the ghost in Stückelberg formulation of nonlinear massive gravity [1619] is missing (for related work, see [20]). This result implies that generally Boulware-Deser ghost is present. On the other hand we show that this additional constraint emerges in two cases when $\omega \neq 0$ and when $\alpha_{\sigma}=0$. We also propose model of consistent extension of the quasidilaton nonlinear massive gravity that can be considered as the generalization of the coupling between massive gravity and the galileon [21]. Then, we argue that given theory is ghost-free, following [19].

This paper is organized as follows. In next Section 2, we review the basic facts about extension of the quasidilaton nonlinear massive gravity as was proposed in [14]. Then, we proceed to the Hamiltonian analysis of given theory and argue that there is no scalar primary constraint that could eliminate the Boulware-Deser ghost. We also discuss the analysis performed recently in [22] and we argue that the constraints introduced there cannot be considered as the gauge fixing constraint. In Section 3, we perform the Hamiltonian analysis of quasidilaton nonlinear massive gravity when we find that there is an additional primary constraint. This result shows that the quasidilaton massive theory as was proposed in [12] is ghost-free at least in their minimal version. Finally in 
Section 4, we propose an extension of the quasidilaton massive gravity that is ghost-free and that can be considered as the generalization of proposal [14]. It would be extremely interesting to analyze cosmological consequences of this theory.

\section{Extension of Quasidilaton Massive Gravity}

In this section, we review basic facts about extension of quasidilaton massive gravity as was proposed in [14]. For simplicity, we restrict ourselves to the minimal form of the massive gravity keeping in mind that its generalization is straightforward.

Explicitly, let us consider the following action:

$$
\begin{gathered}
S=S_{\text {m.g. }}+S_{\sigma}, \\
S_{\text {m.g. }}=M_{p}^{2} \int d^{4} x \sqrt{-\widehat{g}}\left[{ }^{(4)} R+2 m^{2}\left(3-\Omega(\Phi) \sqrt{\widehat{g}^{-1} \tilde{f}}\right)\right],
\end{gathered}
$$

where $\widetilde{f}_{\mu \nu}$ was introduced in [14],

$$
\tilde{f}_{\mu \nu}=f_{\mu \nu}-\frac{\alpha_{\sigma}}{M_{p}^{2} m^{2}} e^{-2 \sigma / M_{p}} \partial_{\mu} \sigma \partial_{\nu} \sigma, \quad f_{\mu \nu}=\partial_{\mu} \phi^{a} \partial_{\nu} \phi^{b} \eta_{a b},
$$

where $\phi^{a}, a, b=0,1,2,3$ are Stückelberg fields and where $\eta_{a b}=\operatorname{diag}(-1,1,1,1)$. Further, $S_{\sigma}$ is defined as

$$
S_{\sigma}=-\frac{\omega}{2} \int d^{4} x \sqrt{-\widehat{g}} \widehat{g}^{\mu \nu} \partial_{\mu} \sigma \partial_{\gamma} \sigma .
$$

Note that $\Omega(\Phi)$ is a function of $\sigma$ which is necessary for the invariance of the theory under global symmetry

$$
\sigma \longrightarrow \sigma+\sigma_{0}, \quad \phi^{a} \longrightarrow e^{-\sigma_{0} / M_{p}} \phi^{a}
$$

so that, under (4), $f_{\mu \nu}$ and $\tilde{f}_{\mu \nu}$ transform as

$$
f_{\mu \nu} \longrightarrow e^{-2 \sigma_{0} / M_{p}} f_{\mu \nu}, \quad \tilde{f}_{\mu \nu} \longrightarrow e^{-2 \sigma_{0} / M_{p}} \widetilde{f}_{\mu \nu} .
$$

The massive term contains square root of the expression $\widehat{g}^{\mu \nu} \tilde{f}_{\nu \rho}$ that, under (4), transforms as

$$
\sqrt{\widehat{\mathfrak{g}}^{-1} \tilde{f}} \longrightarrow e^{-\sigma_{0} / M_{p}} \sqrt{\widehat{\mathfrak{g}}^{-1} \tilde{f}}
$$

which implies that $\Omega(\sigma)$ has to have the form

$$
\Omega(\sigma)=e^{\sigma / M_{p}} .
$$

Now, we are ready to proceed to the Hamiltonian analysis of given theory. Due to the presence of the square root in the action, we perform the redefinition of the shift functions [23, 24]

$$
N^{i}=M \widetilde{n}^{i}+\widetilde{f}^{i k} \widetilde{f}_{0 k}+N \widetilde{D}_{j}^{i} \widetilde{n}^{j},
$$

where

$$
M^{2}=-\widetilde{f}_{00}+\widetilde{f}_{0 k} \widetilde{f}^{k l} \widetilde{f}_{l 0}, \quad \widetilde{f}_{i j} \widetilde{f}^{j k}=\delta_{i}^{j},
$$

and where $\widetilde{D}_{j}^{i}$ obeys the equation

$$
\sqrt{\tilde{x}} \widetilde{D}_{j}^{i}=\sqrt{\left(g^{i k}-\widetilde{D}_{m}^{i} \widetilde{n}^{m} \widetilde{D}_{n}^{k} \widetilde{n}^{n}\right) \widetilde{f}_{k j}}, \quad \tilde{x}=1-\widetilde{n}^{i} \widetilde{f}_{i j} \widetilde{n}^{j}
$$

and also the following important identity:

$$
\widetilde{f}_{i k} \widetilde{D}_{j}^{k}=\widetilde{f}_{j k} \widetilde{D}_{i}^{k} .
$$

We also use $3+1$ decomposition of the four-dimensional metric $\widehat{g}_{\mu \nu}[25,26]$ :

$$
\begin{gathered}
\widehat{g}_{00}=-N^{2}+N_{i} g^{i j} N_{j}, \quad \widehat{g}_{0 i}=N_{i}, \quad \widehat{g}_{i j}=g_{i j}, \\
\widehat{g}^{00}=-\frac{1}{N^{2}}, \quad \widehat{g}^{0 i}=\frac{N^{i}}{N^{2}}, \quad \hat{g}^{i j}=g^{i j}-\frac{N^{i} N^{j}}{N^{2}} .
\end{gathered}
$$

Note that, in $3+1$ formalism, the kinetic term for $\sigma$ has the form

$$
-\frac{\omega}{M_{p}^{2}} \widehat{g}^{\mu \nu} \partial_{\mu} \sigma \partial_{\nu} \sigma=\frac{\omega}{M_{p}^{2}}\left(\nabla_{n} \sigma\right)^{2}-\frac{\omega}{M_{p}^{2}} \partial_{i} \sigma g^{i j} \partial_{j} \sigma,
$$

where, after redefinition (8), $\nabla_{n} \sigma$ has the form

$$
\nabla_{n} \sigma=\frac{1}{N}\left(\partial_{t} \sigma-\left(M \widetilde{n}^{i}+\widetilde{f}^{i k} \widetilde{f}_{0 k}+N \widetilde{D}_{j}^{i} \widetilde{n}^{j}\right) \partial_{i} \sigma\right) .
$$

With the help of these expressions, we rewrite action (1) into the form

$$
\begin{aligned}
S=M_{p}^{2} \int d^{3} \mathbf{x} d t[ & N \sqrt{g} \widetilde{K}_{i j} g^{i j k l} \widetilde{K}_{k l}+N \sqrt{g} R-\sqrt{g} M U- \\
& -2 m^{2}\left(N \Omega(\Phi) \sqrt{g} \sqrt{\tilde{x}} D_{i}^{i}-3 N \sqrt{g}\right)+ \\
& \left.+N \sqrt{g} \frac{\omega}{2 M_{p}^{2}}\left(\nabla_{n} \sigma\right)^{2}-N \sqrt{g} \frac{\omega}{2 M_{p}^{2}} \partial_{i} \sigma g^{i j} \partial_{j} \sigma\right],
\end{aligned}
$$

where

$$
U=2 m^{2} \Omega(\Phi) \sqrt{\widetilde{x}},
$$

and where we used the $3+1$ decomposition of the fourdimensional scalar curvature

$$
{ }^{(4)} R=\widetilde{K}_{i j} \mathscr{G}^{i j k l} \widetilde{K} k l+R,
$$

where $R$ is three-dimensional scalar curvature. We also introduced de Witt metric

$$
\mathscr{G}^{i j k l}=\frac{1}{2}\left(g^{i k} g^{j l}+g^{i l} g^{j k}\right)-g^{i j} g^{k l}
$$

with inverse

$$
\begin{gathered}
\mathscr{G}_{i j k l}=\frac{1}{2}\left(g_{i k} g_{j l}+g_{i l} g_{j k}\right)-\frac{1}{2} g_{i j} g_{k l}, \\
\mathscr{G}_{i j k l} \mathscr{G}^{k l m n}=\frac{1}{2}\left(\delta_{i}^{m} \delta_{j}^{n}+\delta_{i}^{n} \delta_{j}^{m}\right) .
\end{gathered}
$$


Note that, in (17), we ignored the terms containing total derivatives. Finally, note that $\widetilde{K}_{i j}$ is defined as

$$
\widetilde{K}_{i j}=\frac{1}{2 N}\left(\partial_{t} g_{i j}-\nabla_{i} N_{j}(\widetilde{n}, g)-\nabla_{j} N_{i}(\widetilde{n}, g)\right) \text {, }
$$

where $N_{i}$ depends on $\widetilde{n}^{i}$ and $g$ through relation (8).

Now, we could proceed to the Hamiltonian formulation of given theory. However, the structure of the derivative $\nabla_{n} \sigma$ (14) suggests very complicated relations between momenta and velocities. For that reason, we consider simpler case when we presume that $\sigma$ depends on time only. Note that this is the reasonable approximation that does not spoil the physical content of the theory. From (15), we find the momenta conjugate to $N, \widetilde{n}^{i}$, and $g_{i j}$ :

$$
\pi_{N} \approx 0, \quad \pi_{i} \approx 0, \quad \pi^{i j}=M_{p}^{2} \sqrt{g} \mathscr{G}^{i j k l} \widetilde{K}_{k l} .
$$

While, in case of $\phi^{a}$ and $\sigma$, we find

$$
\begin{gathered}
p_{a}=\frac{\mathscr{M}_{a b} \partial_{t} \phi^{b}}{M}\left[\widetilde{n}^{i} \mathscr{R}_{i}+M_{p}^{2} \sqrt{g} U\right]-f^{i j} \partial_{j} \phi_{a} \mathscr{R}_{i}, \\
p_{\sigma}=-\frac{1}{M} \frac{\alpha_{\sigma}}{M_{p}^{2} m^{2}} e^{-2 \sigma / M_{p}^{2}} \partial_{t} \sigma\left(\widetilde{n}^{i} \mathscr{R}_{i}+M_{p}^{2} \sqrt{g} U\right)+\omega \sqrt{g} \partial_{t} \sigma,
\end{gathered}
$$

where

$$
\begin{gathered}
M^{2}=M_{0}^{2}+\frac{\alpha_{\sigma}}{M_{p}^{2} m^{2}} e^{-2 \sigma / M_{p}^{2}}\left(\partial_{t} \sigma\right)^{2}, \quad \mathscr{R}_{i}=-2 g_{i k} \nabla_{j} \pi^{k j}, \\
M_{0}^{2}=-\partial_{t} \phi^{a} \mathscr{M}_{a b} \partial_{t} \phi^{b}, \quad \mathscr{M}_{a b}=\eta_{a b}-\partial_{i} \phi_{a} f^{i j} \partial_{j} \phi_{b} .
\end{gathered}
$$

These relations imply

$$
\begin{aligned}
M_{0}^{2}= & -\frac{1}{\Pi_{a} \mathscr{M}^{a b} \Pi_{b}+\left(\widetilde{n}^{i} \mathscr{R}_{i}+M_{p}^{2} \sqrt{g} U\right)^{2}} \frac{\Pi_{a} \mathscr{M}^{a b} \Pi_{b} \alpha_{\sigma}}{M_{p}^{2} m^{2}} \\
& \times e^{-2 \sigma / M_{p}^{2}}\left(\partial_{t} \sigma\right)^{2},
\end{aligned}
$$

where $\Pi_{a}=p_{a}+f^{i j} \partial_{j} \phi_{a} \mathscr{R}_{i}$. Then, it is easy to find relation between momenta and velocities:

$$
\begin{aligned}
& p_{\sigma}+\sqrt{\frac{\alpha_{\sigma}}{M_{p}^{2} m^{2}}} e^{-\sigma / M_{p}^{2}} \sqrt{\Pi_{a} \mathscr{M}^{a b} \Pi_{b}+\left(\tilde{n}^{i} \mathscr{R}_{i}+M_{p}^{2} \sqrt{g U}\right)^{2}} \\
& =\frac{1}{N} \omega \sqrt{g} \partial_{t} \sigma \\
& \quad \times \frac{\Pi_{a}}{\sqrt{\Pi_{a} \mathscr{M}^{a b} \Pi_{b}+\left(\widetilde{n}^{i} \mathscr{R}_{i}+M_{p}^{2} \sqrt{g} U\right)^{2}}} \\
& =\frac{M_{p} m}{\sqrt{\alpha_{\sigma}}} e^{\sigma / M_{p}^{2}} \mathscr{M}_{a b} \frac{\partial_{t} \phi^{b}}{\partial_{t} \sigma} .
\end{aligned}
$$

It is crucial that these relations do not imply an existence of the scalar primary constraint which is in sharp contrast with the case of the dRGT massive gravity [16] or dRGT massive gravity coupled to the galileon [19]. On the other hand, using the property of the matrix $\mathscr{M}_{a b}$, we find three constraints:

$$
\partial_{i} \phi^{a} \Pi_{a} \equiv \Sigma_{i}=\partial_{i} \phi^{a} p_{a}+\mathscr{R}_{i} \approx 0 .
$$

With suitable extension of these constraints by terms proportional to the primary constraints $\pi_{i} \approx 0$, we find that they are the first class constraints whose smeared forms are the generator of spatial diffeomorphism.

Now, using (25), we determine corresponding Hamiltonian

$$
H=\int d^{3} \mathbf{x} N \mathscr{H}_{0},
$$

where

$$
\begin{aligned}
\mathscr{H}_{0}= & \frac{1}{2 \omega \sqrt{g}}\left(p_{\sigma}+\sqrt{\frac{\alpha_{\sigma}}{M_{p}^{2} m^{2}}} e^{-\sigma / M_{p}^{2}}\right. \\
& \left.\times \sqrt{\Pi_{a} M^{a b} \Pi_{b}+\left(\widetilde{n}^{i} \mathscr{R}_{i}+M_{p}^{2} \sqrt{g} U\right)^{2}}\right)^{2} \\
& +\frac{1}{\sqrt{g} M_{p}^{2}} \pi^{i j} \mathscr{G}_{i j k l} \pi^{k l}-\sqrt{g}(3) R \\
& -2 m^{2}\left(\Omega \sqrt{g} \sqrt{\tilde{x}} \widetilde{D}_{i}^{i}-2 \sqrt{g}\right)+\widetilde{D}_{j}^{i} \tilde{n}^{j} \mathscr{R}_{i} .
\end{aligned}
$$

The requirement of the preservation of the constraint $\pi_{N} \approx$ 0 implies that $\mathscr{H}_{0}$ is a constraint as well. The succeeding analysis is straightforward. We have six first class constraints $\Sigma_{i} \approx 0, \mathscr{H}_{0} \approx 0, \pi_{N} \approx 0$ and six second class constraints $\pi_{i} \approx 0, \mathscr{C}_{i} \approx 0$ where $\mathscr{C}_{i} \approx 0$ are the secondary constraints that arise from the requirement of the preservation of the constraints $\pi_{i} \approx 0$. These constraints can be solved for $\pi_{i}$ and $\widetilde{n}^{i}$. Further, the first class constraint $\pi_{N} \approx 0$ can be gauge fixed that leads to the elimination of $\pi_{N}$ and $N$ as dynamical variables. Finally, the constraints $\Sigma_{i}, \mathscr{H}_{0}$ can be again gauge fixed which leads to the elimination of the Stückelberg fields and conjugate momenta. As a result, we are left with 12 degrees of freedom coming from the gravity sector that can be identified with 10 degrees of freedom corresponding to the massive graviton and two degrees of freedom corresponding to the scalar at least at the linearized approximation. Note that this scalar mode cannot be eliminated due to the absence of the scalar constraint. As a result the Boulware-Deser ghost is generally present. Finally, there are two phase space degrees of freedom $\sigma$ and $p_{\sigma}$.

2.1. Remark about the Paper [22]. After the first version of this paper was published, Mukohyama argued in his paper [22] that the extension of the quasidilaton theory is ghostfree. His arguments is based on the existence of the additional constraints that he imposed on the scalar fields:

$$
\phi^{0}=-e^{-\sigma / M_{p}}, \quad \phi^{i}=\delta_{\mu}^{i} x^{\mu}, \quad i=1,2,3 .
$$

$\mathrm{He}$ called this constraint as the gauge fixing constraint and then he was able to derive the Hamiltonian in the form 
$H=\int d^{3} \mathbf{x} N \mathscr{C}_{0}$, where the specific form of $\mathscr{C}_{0}$ is given in [22]. According to this result, he claimed that there exists an additional constraint in the gauge fixed theory so that this constraint is responsible for the elimination of the BoulwareDeser ghost.

In this section, we reconsider (29) from different point of view and argue that this is not a gauge fixing constraint at all. We do not impose the fixing of spatial diffeomorphism and consider the following relation between $\phi_{0}$ and $\sigma$ (note that we do not use the word "gauge fixing condition"):

$$
\sigma=-M_{p} \ln \phi^{0}
$$

that is equivalent to (29). Inserting this relation to the definition of $\widetilde{f}_{\mu \nu}$, we obtain

$$
\tilde{f}_{\mu \nu}=\eta_{a b} \partial_{\mu} \phi^{a} \partial_{\nu} \phi^{b}-\frac{\alpha_{\sigma}}{m_{g}^{2}} \partial_{\mu} \phi^{0} \partial_{\nu} \phi^{0}
$$

Then, inserting (31) into the dRGT massive gravity, we obtain

$$
\begin{gathered}
S=M_{p}^{2} \int d^{4} x \sqrt{-\widehat{g}}\left[{ }^{(4)} R+2 m^{2}\left(3-\frac{1}{\left(\phi^{0}\right)^{2}} \sqrt{\widehat{g}^{-1} \tilde{f}}\right)\right. \\
\left.-\frac{\omega}{2\left(\phi^{0}\right)^{2}} \widehat{g}^{\mu \nu} \partial_{\mu} \phi^{0} \partial_{\nu} \phi^{0}\right] .
\end{gathered}
$$

Now we see that given action is manifestly diffeomorphism invariant. In other words, while we reduce the number of degrees of freedom by imposing (30), the number of gauge symmetries is the same. However we mean that the fact that we have less degrees of freedom than the original theory while the number of gauge symmetries is the same implies that these two theories have different physical content and should not be considered as equivalent. In other words, condition (29) is not a gauge fixing condition.

From given analysis, it is clear that the constraint $\mathscr{C}_{0}$ that was identified in [22] corresponds to the Hamiltonian constraint in the theory with fixed spatial diffeomorphism. Clearly, this constraint should have vanishing Poisson bracket $\left\{\mathscr{C}_{0}(\mathbf{x}), \mathscr{C}_{0}(\mathbf{y})\right\}$ which implies that it is the first class constraint. Clearly, there is no way how to generate the additional constraint by imposing the requirement of the preservation of the constraint $\mathscr{C}_{0}$ during the time evolution of the system since the Hamiltonian is equal to $H=\int d^{3} \mathbf{x} N \mathscr{C}_{0}$. However, since $\mathscr{C}_{0}$ is the first class constraint, it can be gauge fixed and, hence, we reduce the number of physical degrees of freedom by two. But we should again stress that the theory represented by action (33) is not equivalent to action (1) that represents the extension of the quasidilaton dRGT theory. In summary, we mean that the arguments that were presented in [22] do not prove that the extension of quasidilaton massive gravity is ghost-free.

Let us compare this situation with the imposing static gauge for the Hamiltonian (27). This gauge fixing is represented by four gauge fixed functions:

$$
\mathscr{G}_{0}=\phi^{0}-t \approx 0, \quad \mathscr{G}_{i}=\phi^{i}-x^{i} \approx 0 .
$$

These constraints together with $\mathscr{H}_{0}, \widetilde{\Sigma}_{i}$ form the second class constraints that can be explicitly solved for $p_{a}$. We solve $\mathscr{H}_{0}$ for $p_{0}$ since we can identify the gauge fixed Hamiltonian with $p_{0}=-\mathscr{H}_{\text {g.f. }}$. The resulting Hamiltonian describes the dynamic of the physical degrees of freedom $g_{i j}, \pi^{i j}$, and $\sigma$, $p_{\sigma}$. The detailed counting of the physical degrees of freedom was presented in the end of previous section and we will not repeat it here.

\section{The Case $\alpha_{\sigma}=0$}

In previous section, we saw that the extension of the quasidilaton theory that was suggested in [14] suffers from the presence of Boulware-Deser ghost due to the absence of two additional scalar constraints in the Hamiltonian formulation of given theory. It is instructive to see whether these constraints emerge in the case of quasidilaton massive gravity which corresponds to the situation when $\alpha_{\sigma}=0$. Explicitly, we consider the action $[11,12]$

$$
\begin{aligned}
S=M_{p}^{2} \int d^{3} \mathbf{x} d t[ & N \sqrt{g} \widetilde{K}_{i j} \mathscr{G}^{i j k l} \widetilde{K}_{k l}+N \sqrt{g} R-\sqrt{g} M_{0} U \\
& -2 m^{2}\left(N \sqrt{g} \sqrt{\tilde{x}} \Omega D_{i}^{i}-3 N \sqrt{g}\right) \\
& \left.+N \sqrt{g} \frac{\omega}{M_{p}^{2}}\left(\nabla_{n} \sigma\right)^{2}-N \sqrt{g} \frac{\omega}{M_{p}^{2}} \partial_{i} \sigma g^{i j} \partial_{j} \sigma\right] .
\end{aligned}
$$

Let us now perform the Hamiltonian analysis of action (34). First of all, we find the canonical momenta

$$
\begin{aligned}
& p_{a}=-\left(-\mathscr{M}_{a b} \frac{1}{M_{0}} \partial_{t} \phi^{b} \widetilde{n}^{i}+f^{i j} \partial_{j} \phi_{a}\right) \mathscr{R}_{i} \\
&+\frac{1}{M_{0}} M_{p}^{2} \sqrt{g} \mathscr{M}_{a b} \partial_{t} \phi^{b} U \\
&+\frac{1}{M_{0}} \mathscr{M}_{a b} \partial_{t} \phi^{b} \widetilde{n}^{i} \partial_{i} \sigma p_{\sigma}-\partial_{i} \sigma f^{i k} \partial_{k} \phi_{a} p_{\sigma}, \\
& p_{\sigma}=\omega \sqrt{g} \nabla_{n} \sigma,
\end{aligned}
$$

so that it is easy to find the scalar primary constraint

$$
\begin{aligned}
\Sigma_{p} \equiv & \left(p_{a}+\left(\mathscr{R}_{i}+\partial_{i} \sigma p_{\sigma}\right) f^{i k} \partial_{k} \phi_{a}\right) \eta^{a b} \\
& \times\left(p_{b}+\left(\mathscr{R}_{i}+\partial_{i} \sigma p_{\sigma}\right) f^{i k} \partial_{k} \phi_{b}\right) \\
& +\left(\tilde{n}^{i}\left(\mathscr{R}_{i}+p_{\sigma} \partial_{i} \sigma\right)+M_{p}^{2} \sqrt{g} U\right)^{2} \approx 0,
\end{aligned}
$$

and also, using the fact that $\partial_{i} \phi^{a} \mathscr{M}_{a b}=0$, we find additional three constraints:

$$
\Sigma_{i}=p_{a} \partial_{i} \phi^{a}+\mathscr{R}_{i}+\partial_{i} \sigma p_{\sigma} .
$$

Now, using these constraints, we can simplify (36) so that it has the form

$$
\Sigma_{p}=p_{a} \mathscr{M}^{a b} p_{b}+\left(\widetilde{n}^{i}\left(\mathscr{R}_{i}+p_{\sigma} \partial_{i} \sigma\right)+M_{p}^{2} \sqrt{g} U\right)^{2} \approx 0 .
$$


This has exactly the same form as the scalar constraint that emerges in case of dRGT massive gravity written in the Stückelberg formalism. The minimal form of this gravity was analyzed in [16] and this analysis can be easily applied to our case. From (15), we find the Hamiltonian with all primary constraints included

$$
H_{E}=\int d^{3} \mathbf{x}\left(N \mathscr{C}_{0}+v_{N} \pi_{N}+v^{i} \pi_{i}+\Omega_{p} \Sigma_{p}+\Omega^{i} \widetilde{\Sigma}_{i}\right),
$$

where we introduced the constraint

$$
\tilde{\Sigma}_{i}=\Sigma_{i}+\partial_{i} \tilde{n}^{j} \pi_{j}+\partial_{j}\left(\tilde{n}^{j} \pi_{i}\right)
$$

and where

$$
\begin{aligned}
\mathscr{C}_{0}= & \frac{1}{\sqrt{g} M_{p}^{2}} \pi^{i j} \mathscr{G}_{i j k l} \pi^{k l}-M_{p}^{2} \sqrt{g} R+2 m^{2} M_{p}^{2} \sqrt{g} \sqrt{\tilde{x}} \Omega \widetilde{D}_{i}^{i} \\
& -6 m^{2} M_{p}^{2} \sqrt{g}+\widetilde{D}_{j}^{i} \tilde{n}^{j}\left(\mathscr{R}_{i}+p_{\sigma} \partial_{i} \sigma\right) \\
& +\frac{1}{\sqrt{g} \omega} p_{\sigma}^{2}+\omega \sqrt{g} g^{i j} \partial_{i} \sigma \partial_{j} \sigma .
\end{aligned}
$$

As the next step, we have to perform the analysis of the stability of the primary constraints $\pi_{i} \approx 0, \pi_{N} \approx 0$ and $\Sigma_{p} \approx 0$. In case of the constraint $\pi_{i} \approx 0$, we find

$$
\begin{aligned}
& \partial_{t} \pi_{i}=\left\{\pi_{i}, H\right\}=- {\left[\left(\mathscr{R}_{j}+p_{\sigma} \partial_{j} \sigma\right)-\frac{2 m^{2} M_{p}^{2} \Omega \sqrt{g}}{\sqrt{\tilde{x}}} n^{k} f_{k j}\right] } \\
& \times {\left[N \frac{\delta\left(\widetilde{D}_{m}^{j} \widetilde{n}^{m}\right)}{\delta \widetilde{n}^{i}}+\delta_{i}^{j}\left(\widetilde{n}^{i}\left(\mathscr{R}_{i}+p_{\sigma} \partial_{i} \sigma\right)\right.\right.} \\
&\left.\left.+M_{p}^{2} \sqrt{g} U\right)\right]=0
\end{aligned}
$$

so that we impose the following secondary constraint

$$
\mathscr{C}_{i}=\mathscr{R}_{i}+p_{\sigma} \partial_{i} \sigma-\frac{2 m^{2} M_{p}^{2} \Omega \sqrt{g}}{\sqrt{\tilde{x}}} f_{i j} \tilde{n}^{j}
$$

Now, with the help of this constraint and the constraint $\widetilde{\Sigma}_{i}$, we can simplify $\Sigma_{p}$ in a similar way as in [16]:

$$
\Sigma_{p}=4 m^{4} M_{p}^{4} g \Omega^{2}+p_{A} \eta^{A B} p_{B} .
$$

Then, it is easy to show that $\left\{\Sigma_{p}(\mathbf{x}), \Sigma_{p}(\mathbf{y})\right\}=0$ and the requirement of the preservation of given constraint leads to the emergence of an additional constraint. These constraints are responsible for the elimination of Boulware-Deser ghost; see again [16] for more details. In other words, the presence of the kinetic term for the quasidilaton that minimally couples to gravity does not spoil the property that the given theory is ghost-free.

\section{Ghost-Free Extension of Quasidilaton Massive Gravity}

We argued, in Section 2, that the extension of quasidilaton theory as was formulated in [14] is plagued by the presence of the Boulware-Deser ghost. On the other hand, given theory has many nice properties so that it is desirable to propose its ghost-free version. In this section, we propose such a formulation when we replace the kinetic term for $\sigma$ by following tadpole galileon term:

$$
S_{\sigma}=-T \int d^{4} x \Psi\left(\Phi^{A}\right) \sqrt{-\operatorname{det} \tilde{f}_{\mu \nu}}=-T \int d^{4} x \Psi\left(\Phi^{A}\right) M \sqrt{\tilde{f}},
$$

where $\Phi^{A}=\left(\phi^{a}, \sigma\right), \tilde{f}=\operatorname{det} \tilde{f}_{i j}$ and where the function $\Psi\left(\Phi^{A}\right)$ was chosen in such a way that action (45) is invariant under (4). We claim that the quasidilaton theory formulated as the dRGT massive theory with $\tilde{f}_{\mu \nu}$ and with the kinetic term for the galileon given by (45) is ghost-free.

To see this explicitly, it is useful to introduce the following notation. Let s write $\widetilde{f}_{\mu \nu}$ as

$$
\tilde{f}_{\mu \nu}=\partial_{\mu} \Phi^{A} \mathscr{G}_{A B} \partial_{\nu} \Phi^{B}
$$

where we introduced the metric $\mathscr{G}_{A B}$ :

$$
\mathscr{G}_{A B}=\left(\begin{array}{cc}
\eta_{A B} & 0 \\
0 & -\frac{\alpha_{\sigma}}{M_{p}^{2} m^{2}} e^{-2 \sigma / M_{p}}
\end{array}\right) .
$$

We see that our proposal has the form of the galileon coupled to dRGT massive gravity [21] whose Hamiltonian analysis was performed in [19]. On the other hand, the action defined by (45) is more complicated since the metric $\eta_{A B}$ is replaced with the more general metric $\mathscr{G}_{A B}$ that depends on $\Phi^{A}$ and there are also additional scalar fields $\Omega\left(\phi^{A}\right), \Psi\left(\Phi^{A}\right)$. However, we can expect that this fact will not modify the constraint structure of given theory.

To see this explicitly, let us briefly review the Hamiltonian analysis of the nonlinear massive gravity with the term (45) keeping in mind that more detailed analysis can be found in [19]. As usual, the momenta conjugate to $N, \widetilde{n}^{i}$, and $g_{i j}$ are

$$
\pi_{N} \approx 0, \quad \pi_{i} \approx 0, \quad \pi^{i j}=M_{p}^{2} \sqrt{g} \mathscr{G}^{i j k l} \widetilde{K}_{k l}
$$

while the momentum conjugate to $\Phi^{A}$ has the form

$$
p_{A}=-\left(\frac{\delta M}{\delta \partial_{t} \Phi^{A}} \tilde{n}^{i}+\mathscr{G}_{A B} \tilde{f}^{i j} \partial_{j} \Phi^{B}\right) \mathscr{R}_{i}-M_{p}^{2} \sqrt{g} \frac{\delta M}{\partial_{t} \Phi^{A}} U^{\prime}
$$

where

$$
U^{\prime}=2 m^{2} \Omega(\Phi) \sqrt{\tilde{x}}+\frac{T}{M_{p}^{2}} \Psi(\Phi) \sqrt{\tilde{f}}
$$

and where $M^{2}$ has the form

$$
\begin{aligned}
& M^{2}=-\partial_{t} \Phi^{A} \mathscr{M}_{A B} \partial_{t} \Phi^{B} \\
& \mathscr{M}_{A B}=\mathscr{G}_{A B}-\mathscr{G}_{A C} \partial_{i} \Phi^{C} \tilde{f}^{i j} \partial_{j} \Phi^{D} \mathscr{G}_{D B} .
\end{aligned}
$$


Note that the matrix $\mathscr{M}_{A B}$ obeys following relations:

$$
\mathscr{M}_{A B} \mathscr{G}^{B C} \mathscr{M}_{C D}=\mathscr{M}_{A D}, \quad \partial_{i} \Phi^{A} \mathscr{M}_{A B}=0 .
$$

Then, it is easy to determine the following primary constraints:

$$
\begin{gathered}
\Sigma_{p}=\left(\widetilde{n}^{i} \mathscr{R}_{i}+M_{p}^{2} \sqrt{g} U^{\prime}\right)^{2}+\left(p_{A}+\mathscr{R}_{i} \widetilde{f}^{i j} \mathscr{G}_{A C} \partial_{j} \Phi^{C}\right) \mathscr{G}^{A B} \\
\times\left(p_{B}+\mathscr{R}_{i} \widetilde{f}^{i j} \mathscr{G}_{B D} \partial_{j} \Phi^{D}\right) \approx 0 \\
\partial_{i} \Phi^{A} p_{A}+\mathscr{R}_{i}=\Sigma_{i} \approx 0
\end{gathered}
$$

Now, we are ready to write the extended Hamiltonian which includes all the primary constraints:

$$
H_{E}=\int d^{3} \mathbf{x}\left(N \mathscr{C}_{0}+v_{N} \pi_{N}+v^{i} \pi_{i}+\Omega_{p} \Sigma_{p}+\Omega^{i} \widetilde{\Sigma}_{i}\right),
$$

where

$$
\begin{aligned}
\mathscr{C}_{0}= & \frac{1}{\sqrt{g} M_{p}^{2}} \pi^{i j} \mathscr{G}_{i j k l} \pi^{k l}-M_{p}^{2} \sqrt{g} R \\
& +2 m^{2} M_{p}^{2} \sqrt{g} \Omega(\Phi) \sqrt{\tilde{x}} \widetilde{D}_{i}^{i}-6 m^{2} M_{p}^{2} \sqrt{g}+\widetilde{D}_{j}^{i} \widetilde{n}^{j} \mathscr{R}_{i}
\end{aligned}
$$

and where we introduced the constraints $\widetilde{\Sigma}_{i}$ defined as

$$
\widetilde{\Sigma}_{i}=\Sigma_{i}+\partial_{i} \widetilde{n}^{i} \pi_{i}+\partial_{j}\left(\tilde{n}^{j} \pi_{i}\right)
$$

To proceed further, we have to check the stability of all constraints. The procedure is the same as in [19] so that we find that $\widetilde{\Sigma}_{i}$ are the first class constraints while the requirement of the preservation of the constraints $\pi_{i} \approx 0$ implies following secondary constraints $[23,24]$ :

$$
\mathscr{C}_{i} \equiv \mathscr{R}_{i}-\frac{2 m^{2} M_{p}^{2} \Omega(\Phi) \sqrt{g}}{\sqrt{\tilde{x}}} \tilde{f}_{i j} \tilde{n}^{j} \approx 0 .
$$

Further, the requirement of the preservation of the constraint $\pi_{N} \approx 0$ implies an existence of the secondary constraint $\mathscr{C}_{0} \approx$ 0 . Using the constraints $\mathscr{C}_{i}$ and $\Sigma_{i}$, we replace the constraint $\Sigma_{p}$ by new independent constraint $\widetilde{\Sigma}_{p}$ :

$$
\begin{aligned}
\tilde{\Sigma}_{p}= & 4 m^{4} M_{p}^{4} \Omega^{2} g+p_{A} \mathscr{G}^{A B} p_{B}+2 T \Psi \sqrt{\tilde{f}} \\
& \times \sqrt{p_{A} \partial_{i} \phi^{A} \tilde{f}^{i j} \partial_{j} \phi^{B} p_{B}+4 m^{4} M_{p}^{4} \Omega^{2} g}+T^{2} \Psi^{2} \tilde{f}=0 .
\end{aligned}
$$

Then, the total Hamiltonian with all constraints included has the form

$$
H_{T}=\int d^{3} \mathbf{x}\left(N \mathscr{C}_{0}+v_{N} \pi_{N}+v^{i} \pi_{i}+\Omega_{p} \widetilde{\Sigma}_{p}+\Omega^{i} \widetilde{\Sigma}_{i}+\Gamma^{i} \mathscr{C}_{i}\right) .
$$

Now, we are ready to analyze the stability of all constraints that appear in (59). Again, the analysis is the same as in [19] with slight complication that now there are additional terms $\Psi(\Phi), \Omega(\Phi)$ together with $\mathscr{G}_{A B}\left(\Phi^{A}\right)$ in the definition of the action. However, these terms are local functions of $\Phi^{A}$ so that they do not affect the result that $\left\{\widetilde{\Sigma}_{p}(\mathbf{x}), \widetilde{\Sigma}_{p}(\mathbf{y})\right\} \approx$ 0 . As a result, the requirement of the preservation of the constraint $\widetilde{\Sigma}_{p} \approx 0$ implies new constraint $\widetilde{\Sigma}_{p}^{I I} \approx 0$. These two constraints are the second class constraints that can be used for the elimination of the Boulware-Deser ghost mode and its conjugate momenta.

In this section, we proposed an extension of the quasidilaton massive gravity that is ghost-free. This proposal can be generalized in different ways, either consider the most general potential of the dRGT massive gravity or more complicated kinetic term for $\sigma$. It would be also very interesting to analyze the cosmological consequences of the model with action (45) following [14].

\section{Conflict of Interests}

The author declares that there is no conflict of interests regarding the publication of this paper.

\section{Acknowledgment}

This work was supported by the Grant agency of the Czech republic under the Grant P201/12/G028.

\section{References}

[1] C. de Rham and G. Gabadadze, "Generalization of the FierzPauli action," Physical Review D, vol. 82, Article ID 044020, 2010.

[2] C. de Rham, G. Gabadadze, and A. J. Tolley, "Resummation of massive gravity," Physical Review Letters, vol. 106, Article ID 231101, 2011.

[3] D. G. Boulware and S. Deser, "Can gravitation have a finite range?" Physical Review D, vol. 6, no. 12, pp. 3368-3382, 1972.

[4] D. G. Boulware and S. Desser, "Inconsistency of finite range gravitation," Physics Letters B, vol. 40, no. 2, pp. 227-229, 1972.

[5] A. de Felice, A. E. Gümrükçüoğlu, and S. Mukohyama, "Massive gravity: non-linear instability of the homogeneous and isotropic universe," Physical Review Letters, vol. 109, Article ID 171101, 2012.

[6] K. Koyama, G. Niz, and G. Tasinato, "The self-accelerating universe with vectors in massive gravity," Journal of High Energy Physics, vol. 2011, no. 12, article 65, 2011.

[7] G. Tasinato, K. Koyama, and G. Niz, "Vector inst abilities and self-acceleration in the decoupling limit of massive gravity," Physical Review D, vol. 87, Article ID 064029, 2013.

[8] N. Khosravi, G. Niz, K. Koyama, and G. Tasinato, "Stability of the self-accelerating universe in massive gravity," Journal of Cosmology and Astroparticle Physics, vol. 2013, no. 8, p. 44, 2013.

[9] G. D’Amico, C. de Rham, S. Dubovsky, G. Gabadadze, D. Pirtskhalava, and A. J. Tolley, "Massive cosmologies," Physical Review D, vol. 84, Article ID 124046, 2011.

[10] A. E. Gümrükçüoğlu, C. Lin, and S. Mukohyama, "Anisotropic Friedmann-Robertson-Walker universe from nonlinear massive gravity," Physics Letters B, vol. 717, no. 4-5, pp. 295-298, 2012. 
[11] A. de Felice, A. E. Gümrükçüoğlu, C. Lin, and S. Mukohyama, "Nonlinear stability of cosmological solutions in massive gravity," Journal of Cosmology and Astroparticle Physics, vol. 1305, no. 5, article 035, 2013.

[12] G. D’Amico, G. Gabadadze, L. Hui, and D. Pirtskhalava, "Quasidilaton: theory and cosmology," Physical Review D, vol. 87, Article ID 064037, 2013.

[13] Q.-G. Huang, Y.-S. Piao, and S.-Y. Zhou, "Mass-varying massive gravity," Physical Review D, vol. 86, Article ID 124014, 2012.

[14] A. de Felice and S. Mukohyama, "Towards consistent extension of quasidilaton massive gravity," Physics Letters B, vol. 728, pp. 622-625, 2014.

[15] G. Gabadadze, K. Hinterbichler, J. Khoury, D. Pirtskhalava, and M. Trodden, "Covariant master theory for novel Galilean invariant models and massive gravity," Physical Review D: Particles, Fields, Gravitation and Cosmology, vol. 86, no. 12, Article ID 124004, 2012.

[16] J. Kluson, "Note about Hamiltonian formalism for general nonlinear massive gravity action in Stuckelberg formalism," International Journal of Modern Physics A: Particles and Fields. Gravitation. Cosmology, vol. 28, Article ID 1350160, 16 pages, 2013.

[17] J. Klusoň, "Non-linear massive gravity with additional primary constraint and absence of ghosts," Physical Review D, vol. 86, Article ID 044024, 2012.

[18] S. F. Hassan, A. Schmidt-May, and M. von Strauss, "Proof of consistency of nonlinear massive gravity in the Stückelberg formulation," Physics Letters B, vol. 715, pp. 355-339, 2012.

[19] J. Kluson, "Hamiltonian analysis of minimal massive gravity coupled to Galileon tadpole term," Journal of High Energy Physics, vol. 2013, article 80, 2013.

[20] Q. G. Huang, K. C. Zhang, and S. Y. Zhou, "Generalized massive gravity in arbitrary dimensions and its Hamiltonian formulation," Journal of Cosmology and Astroparticle Physics, vol. 1308, article 050, 2013.

[21] M. Andrews, G. Goon, K. Hinterbichler, J. Stokes, and M. Trodden, "Massive gravity coupled to Galileons is ghost-free," Physical Review Letters, vol. 111, no. 6, Article ID 061107, 2013.

[22] S. Mukohyama, "Extended quasidilaton massive gravity is ghost free," http://arxiv.org/abs/1309.2146.

[23] S. F. Hassan and R. A. Rosen, "On non-linear actions for massive gravity," Journal of High Energy Physics, vol. 2011, article 9, 2011.

[24] S. F. Hassan and R. A. Rosen, "Resolving the ghost problem in non-linear massive gravity," Physical Review Letters, vol. 108, Article ID 041101, 2012.

[25] E. Gourgoulhon, " $3+1$ formalism and bases of numerical relativity," http://arxiv.org/abs/gr-qc/0703035.

[26] R. L. Arnowitt, S. Deser, and C. W. Misner, "The dynamics of general relativity," http://arxiv.org/abs/grqc/0405109. 

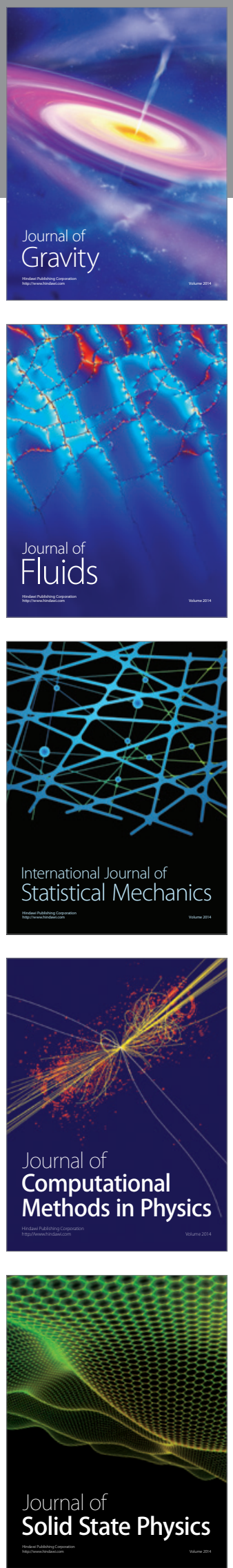

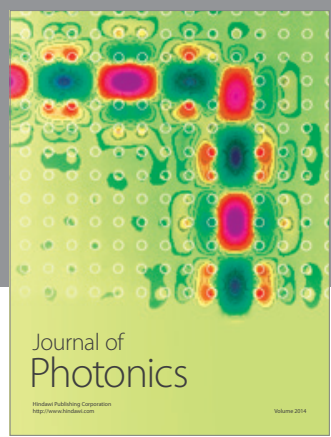

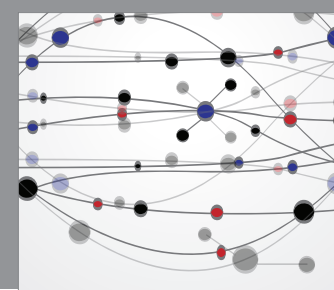

The Scientific World Journal

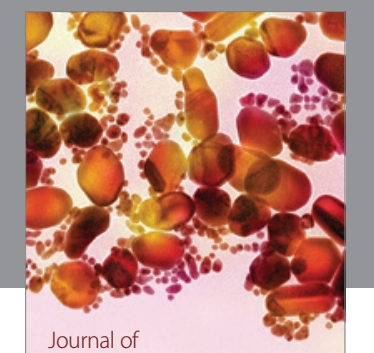

Soft Matter
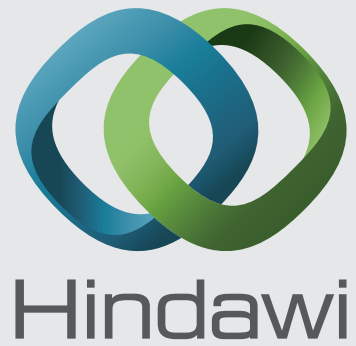

Submit your manuscripts at

http://www.hindawi.com
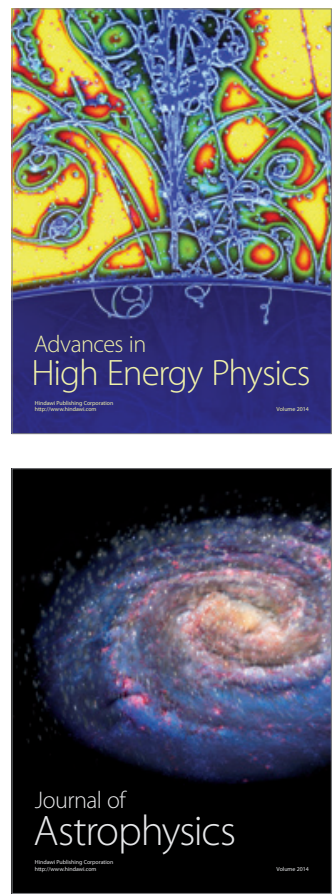
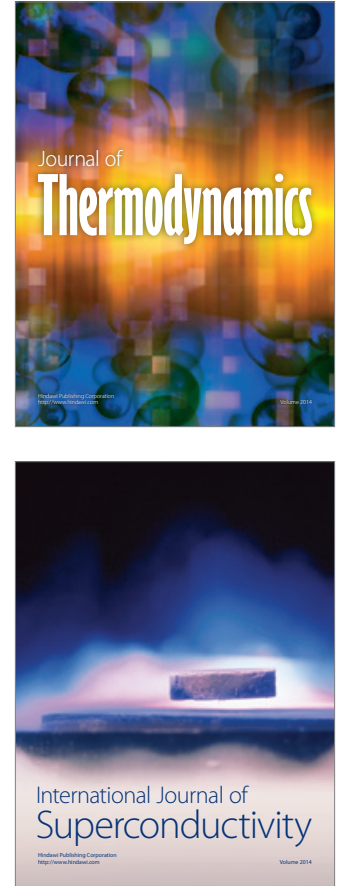
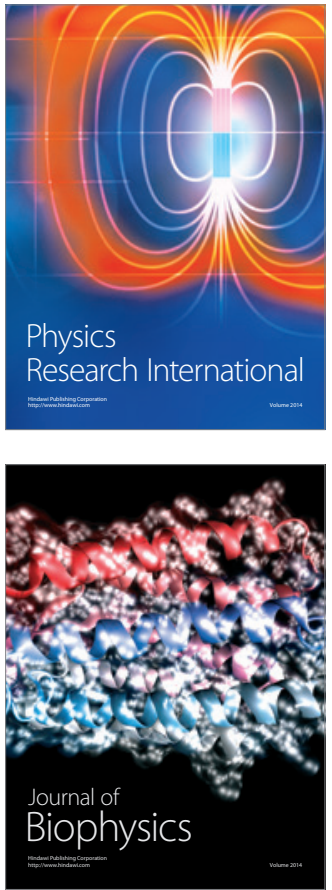
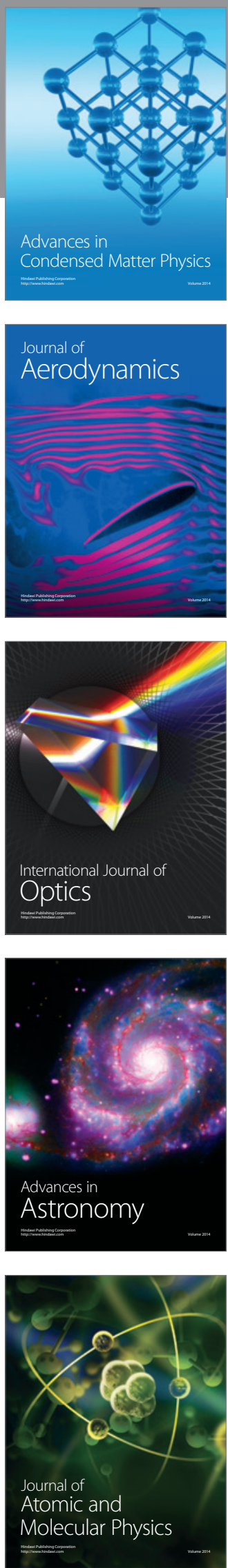